\title{
A REPRESENTATION THEOREM FOR DIFFERENTIABLE FUNCTIONS
}

\author{
VŨ TRỌNG TUẤN AND ĐÃNG ĐìNH ÁNG
}

\begin{abstract}
The authors give a representation formula for differentiable functions analogous to Taylor's formula, but in which the differentiability of the remainder term is recovered. The result is used to prove a generalization of the Morse lemma.
\end{abstract}

In this paper we give a representation formula for differentiable functions analogous to Taylor's formula, but in which the differentiability of the remainder term is recovered. Our result can be considered as an infinite dimensional version of Whitney's theorem on the differentiability of the remainder term in Taylor's formula for the finite dimensional case.

The paper is divided into two sections. In $\S 1$, we prove a representation theorem for differentiable functions, and a converse of the latter. In $\$ 2$, we shall illustrate the usefulness of the foregoing results by using them to give a very simple proof of Kuiper's refinement of Palais' Morse lemma for Hilbert spaces; in fact, what we prove is a generalization of Kuiper's result.

\section{A representation of differentiable functions.}

1.1. Let $f$ be of class $C^{n+k}, 1 \leqslant n<\infty, 0 \leqslant k \leqslant \infty$, in an open convex neighborhood $\theta$ of 0 in a normed linear space with values in a Banach space. Then, by Taylor's theorem,

$$
f(x)=\sum_{j=0}^{n}\left(D^{j} f(0) / j !\right) x^{(j)}+R_{n}(x) x^{(n)}
$$

in which

$$
R_{n}(x)=\int_{0}^{1}\left((1-t)^{n-1} /(n-1) !\right)\left(D^{n} f(t x)-D^{n} f(0)\right) d t .
$$

Here $R_{n}(x)$ is an $n$-linear mapping, and $R_{n}$ is of class $C^{k}$ but usually not more. We shall give below a representation, which will be a useful substitute for Taylor's expansion in the sense that the differentiability of the remainder term is recovered (cf. Theorem 1). Our functions will be defined on open subsets of a normed linear space $V$ having a $C^{r}$ duality mapping in the following sense:

Received by the editors November 3, 1977 and, in revised form, August 15, 1978.

AMS (MOS) subject classifications (1970). Primary 57D35; Secondary 58E05.

Key words and phrases. Whitney's theorem on differentiability of the remainder term in Taylor's formula, Morse lemma. 
There is a $C^{r}$ mapping $e$ of $V \backslash\{0\}$ into the topological dual $V^{*}$ of $V$ such that $e(x) x=1$ for every $x \neq 0$ in $V$

and

$$
\begin{aligned}
& \|x\|^{i+1} D^{i} e(x) \text { is bounded in a neighborhood of } 0 \text { for } \\
& 0 \leqslant i \leqslant r .
\end{aligned}
$$

Note that every inner product space has a $C^{\infty}$ duality mapping and that so does every Sobolev space $W^{p, k}(\Omega)$ for even $p$.

We can now state

1.2. Theorem 1. Suppose $V$ is a normed linear space having a $C^{n+k}$ duality mapping and $f: \theta \leadsto F$ is a $C^{n+k}$ mapping from an open neighborhood $\theta$ of 0 in $V$ into a Banach space $F$. Then there is a mapping $S_{n}: \theta \leadsto L_{s}^{n}(V ; F)$ (the Banach space of bounded symmetric n-linear mappings from $V^{n}$ to $F$ ) with the following properties:

(i) $S_{n}$ is $C^{k}$ in $\theta, S_{n}(0)=0$ and

$$
D^{i} S_{n}(0)=(i ! /(n+i) !) D^{n+i} f(0) \text { for } 1<i<k \text {. }
$$

(ii)

$$
S_{n} \text { is } C^{n+k} \text { in } \theta \backslash\{0\}
$$

and

$$
\lim _{x \rightarrow 0}\|x\|^{i} D^{i+k} S_{n}(x)=0 \text { for } 1<i<n .
$$

(iii) In $\theta, f$ is represented by

$$
f(x)=\sum_{j=0}^{n}\left(D^{j} f(0) / j !\right) x^{(j)}+S_{n}(x) x^{(n)} .
$$

REMARK 1. The mapping $S_{n}$ depends on the choice of the duality mapping and is usually not unique.

REMARK 2. In the one dimensional case, the representation formula (5) is nothing but Taylor's formula. Whitney [9] established the properties (3), (4b), (5) of Theorem 1 for $S_{n}=R_{n}$ in the one dimensional case. He also gave a representation formula different from Taylor's formula in the higher finite dimensional case, but even in this case, our results are useful complements to his. Note that (4a) is trivial in the one dimensional case, but not so in the general, even finite dimensional, case. Assertion (i) shows that $R_{n}$ and $S_{n}$ have the same derivatives at 0 up to order $k$.

REMARK 3. Note that (3) is equivalent to

$$
\lim _{x \rightarrow 0} D^{i} S_{n}(x)=(1 /(n+i) !) D^{n+i} f(0) \text {. }
$$

The latter remark will be used later.

In the case $k=\infty, R_{n}$ satisfies all assertions of Theorem 1, so it remains to consider the case $k<\infty$. 
Before proving Theorem 1, we give below its converse which is useful in proving that certain functions are $C^{r}$.

THeOREM 2. Let $V$ be a normed linear space, let $\theta$ be an open neighborhood of 0 in $V$ and let $S: \theta \leadsto L^{n}(V ; F)$ be a mapping of $\theta$ into the Banach space of bounded $n$-linear mappings from $V^{n}$ into a Banach space $F$. Suppose

(i) $S$ is $C^{k}$ in $\theta$,

(ii) $S$ is $C^{n+k}$ in $\theta \backslash\{0\}$ and

$$
\lim _{x \rightarrow 0}\|x\|^{i} D^{i+k} S(x)=0 \text { for } 1<i<n .
$$

Let $g(x)=S(x) x^{(n)}$. Then $g$ is of class $C^{n+k}$ in $\theta$.

REMARK 4. For different versions of converses to Taylor's theorem, cf. Abraham and Robbin [1].

1.3. Let $\alpha$ denote a $p$-tuple $\left(\alpha_{1}, \ldots, \alpha_{p}\right)$ of nonnegative integers. For $|\alpha|=i$, consider an $\alpha$-partition $\sigma$, that is a partition of $\{1, \ldots, i\}$ into subsets $\sigma_{1}, \ldots, \sigma_{p}$ (some of which may be empty) having $\alpha_{1}, \ldots, \alpha_{p}$ elements respectively, with the understanding that

$$
\sigma=\sigma^{\prime} \text { if and only if } \sigma_{1}=\sigma_{1}^{\prime}, \ldots, \sigma_{p}=\sigma_{p}^{\prime} .
$$

Note that a given partition of $\{1, \ldots, i\}$ may give rise to more than one $\alpha$-partition. An $\alpha$-partition $\sigma$ will usually be denoted by $\sigma(\alpha)$.

An $i$-tuple $\left(h_{1}, \ldots, h_{i}\right)$ of vectors is denoted by $h$, the sub-tuple with subscripts in $\sigma_{j}$ is denoted by $h^{\sigma j}$.

We shall repeatedly use the following Leibnitz formula:

Let $P$ be a bounded p-linear mapping and let $f_{1}, \ldots, f_{p}$ be $C^{r}$ mappings. Let

$$
g(x)=P\left(f_{1}(x), \ldots, f_{p}(x)\right)=P\left(\left(f_{j}(x)\right)_{j=1, \ldots, p}\right) .
$$

Then the derivatives of $g$ are given by

$$
D^{i} g(x) h=\sum_{|\alpha|=i} \sum_{\sigma(\alpha)} P\left(\left(D^{\alpha_{j}} f_{j}(x) h^{\sigma_{j}}\right)_{j=1, \ldots, p}\right)
$$

where the first sum is over all the $\sigma_{j}$ 's in a given $\alpha$-partition $\sigma(\alpha)$ with $|\alpha|=i$ and the second sum is over all the $\alpha$-partitions $\sigma(\alpha)$ with $|\alpha|=i$.

We now turn to the proofs of Theorems 1 and 2 successively.

Proof OF THEOREM 1.

1.4. Let $f$ be as in Theorem 1 , let $0<k<\infty$, and put $r=n+k$. Let

$$
g_{r}(x)=f(x)-\sum_{j=0}^{r}\left(D^{j} f(0) / j !\right) x^{(j)}
$$

Then $g_{r}$ is $C^{r}$ and $D^{i} g_{r}(0)=0$ for $i=0,1, \ldots, r$. Hence by Taylor's formula applied to $D^{i} g_{r}$,

$$
\lim _{x \rightarrow 0}\|x\|^{i-r} D^{i} g_{r}(x)=0, \quad 0<i<r
$$


Define $S_{r}: \theta \rightarrow L_{s}^{r}(V ; F)$ by

$$
S_{r}(0)=0 \text { and } S_{r}(x)=(\otimes r e(x)) \otimes g_{r}(x) \text { for } x \neq 0 .
$$

The notation in (8) means

$$
S_{r}(x)\left(x_{1}, \ldots, x_{r}\right)=\left(\prod_{i=1}^{r} e(x) x_{i}\right) g_{r}(x) \text { for }\left(x_{1}, \ldots, x_{r}\right) \text { in } V^{r} \text {. }
$$

Clearly $S_{r}(x)$ is a bounded $r$-linear symmetric mapping. Note that $S_{r}$ is the composition of a bounded $(r+1)$-linear map

$$
P:\left(V^{*}\right)^{r} \times F \leadsto L^{r}(V ; F)
$$

with the $C^{r}$-mapping

$$
x \leadsto\left((e(x))^{(r)}, g_{r}(x)\right) .
$$

Hence by Leibnitz' formula,

$$
D^{i} S_{r}(x) h=\sum_{|\alpha|=i} \sum_{\sigma(\alpha)} P\left(\left(D^{\alpha_{j}} e(x) h^{\sigma_{j}}\right)_{j=1, \ldots, r}, D^{\alpha_{r+1}} g_{r}(x) h^{\sigma_{r+1}}\right) .
$$

By (2), (7) and (9), we have

$$
\lim _{x \rightarrow 0}\|x\|^{i} D^{i} S_{r}(x)=0 \text { for } 0<i<r .
$$

For $1<n<r$, put

$S_{n}(x)=S_{r}(x) x^{(k)}+\sum_{j=1}^{k}\left(D^{n+j} f(0) /(n+j) !\right) x^{(j)} \equiv U_{n}(x)+V_{n}(x)$.

Then $S_{n}$ satisfies (4a) and (5). The proof of the theorem will be completed once it is shown that $S_{n}$ satisfies (3) and (4b). Now, $V_{n}$ is $C^{\infty}$ and

$$
\begin{aligned}
V_{n}(0) & =0, \\
D^{i} V_{n}(0) & =(i ! /(n+i) !) D^{n+i} f(0), \quad i<i<k, \\
D^{j+k} V_{n}(x) & =0, \quad 1<j<n .
\end{aligned}
$$

Consider next $U_{n}(x)$. Note that $U_{n}$ is $C^{k}$ in $\Theta$ by Theorem 2 since $S_{r}$ is $C^{0}$ in $\theta$. Moreover, we have by Leibnitz' formula:

$$
\begin{aligned}
D^{i} U_{n}(x) h & =\sum_{|\alpha|=i} \sum_{\sigma(\alpha)} D^{\alpha_{1}} S_{r}(x) h^{\sigma_{1}}\left(D^{\alpha_{j} \mathrm{id}}(x) h^{\sigma_{j}}\right)_{j=2, \ldots, k+1} \\
& \quad \text { (id: the identity map of } V) \\
& \equiv A_{i}(x) h .
\end{aligned}
$$

By (10), $\lim \|x\|^{\alpha_{1}} D^{\alpha_{1}} S_{r}(x)=0$ for $x \rightarrow 0$. Since $\|x\|^{j-1} D^{j \mathrm{id}(x)}$ is bounded near 0 , we have for $x \leadsto 0$ :

$$
\lim A_{i}(x)=0, \quad 0<i<k,
$$


and

$$
\lim \|x\|^{j} A_{j+k}(x)=0, \quad 1<j<n .
$$

(12a), (12b) and (14) together imply in view of Remark 3 that $S_{n}$ satisfies (3). Furthermore, (12c) and (15) together imply that $S_{n}$ satisfies (4b). This completes the proof of the theorem.

We now turn to

Proof of Theorem 2. We have $S(x) x^{(n)}=\left(S(x) x^{(n-1)}\right) x$, hence Theorem 2 can be proved by induction on $n$; in fact, it can be reduced to the case $n=1$.

Let $g(x)=S(x) x$, we have by Leibnitz' formula

$$
\begin{aligned}
D^{1+k} g(x) h & =\left(D^{1+k} S(x) h\right) x+\sum_{j_{0}=1}^{k+1} D^{k} S(x)\left(\left(h_{j}\right)_{j \neq j_{0}}\right) h_{j_{0}} \\
& =A(x) h .
\end{aligned}
$$

By hypothesis (ii), $A(x)$ converges to $A$ as $x \leadsto 0$, where

$$
A h=\sum_{j_{0}=1}^{k+1} D^{k} S(0)\left(\left(h_{j}\right)_{j \neq j_{0}}\right) h_{j_{0}} .
$$

Hence, $D^{1+k} g(0)$ exists by Remark 3, and furthermore $g$ is $C^{1+k}$. This completes the proof of Theorem 2.

2. The Morse lemma. The Morse lemma was extended to the infinite dimensional case by Palais ([4] and [5]; cf. also [2] for an elementary proof). Palais' result was generalized by several authors, among whom we mention Tromba [6], [7], Kuiper [3] to quote a few (Kuiper's manuscript was kindly made available to us by G. Barbançon, University of Strasbourg).

We shall use Theorems 1 and 2 in the proof of a generalized Morse lemma for Banach spaces having smooth duality mappings. The present version of the Morse lemma can be considered an extension of Kuiper's result. Note also that our proof is considerably simpler than Kuiper's proof, even for the Hilbert space case.

We shall say after Palais, that 0 is a nondegenerate critical point of $f: \theta \rightarrow \mathbf{R}$ if $D f(0)=0$ and $D^{2} f(0)$ is an isomorphism of $V$ onto $V^{*}$.

We now state and prove

THEOREM 3. Let $V$ be a Banach space having a $C^{2+k}$ duality mapping, $\theta$ an open neighborhood of 0 in $V$, and

$$
f: \mathcal{O} \leadsto \mathbf{R}
$$

a mapping of class $C^{2+k}(k \geqslant 0)$ admitting 0 as a nondegenerate critical point with $f(0)=0$. Then there exist a neighborhood $U$ of 0 and a diffeomorphism $\varphi$ of $U$ into $\theta$ such that

(i) $\varphi$ is $C^{1+k}$ in $U$ and $C^{2+k}$ in $U \backslash\{0\}$,

(ii) $\varphi(0)=0, D \varphi(0)=\mathrm{id}_{V}$ (the identity mapping of $V$ ),

(iii) $f(\varphi(x))=\frac{1}{2} D^{2} f(0) x^{(2)},(x$ in $U)$. 
Proof. 0 is a nondegenerate critical point of $f$, hence $D f(0)=0$ and $D^{2} f(0)$ is an isomorphism of Banach spaces. Put $B=\frac{1}{2} D^{2} f(0)$. By the representation formula (Theorem 1),

$$
f(x)=\left(B+S_{2}(x)\right) x^{(2)}, \quad(x \text { in } \theta),
$$

where $S_{2}$ satisfies (i)-(iii) of Theorem 1 with $n=2$.

In this paragraph we establish the existence of a local diffeomorphism $\varphi$ satisfying (i)-(iii) of the theorem.

Put

$$
S(x)=B^{-1} \circ\left(S_{2}(x)\right), \quad F(x)=\mathrm{id}_{V}+S(x) .
$$

Then

$$
f(x)=B(F(x) x) x
$$

Let

$$
E=\{h \text { in } L(V ; V): B(h(x)) y=B(h(y)) x \text { for every } x \text { and } y \text { in } V\}
$$

and

$$
T: E \leadsto L_{s}(V ; V), \quad T h=B \circ h,
$$

where $L(V ; V)=L^{1}(V ; V)$ and $L_{s}(V ; V)=L_{s}^{1}(V ; V)$.

It is clear that $E$ is a closed subspace of $L(V ; V)$ and that $T$ is an isomorphism of Banach spaces.

For $h_{1}$ and $h_{2}$ in $E$ we have

$$
B\left(h_{1} \circ h_{2}(x)\right) y=B\left(h_{2} \circ h_{1}(y)\right) x, \quad(x, y \text { in } V),
$$

so $h \in E$ implies $h^{n} \in E$ for $n=2,3, \ldots$ Here $h^{2}=h \circ h$ and $h^{n}=$ $h^{n-1} \circ h$. Let $\theta: E \leadsto E, \theta(u)=u^{2}$. Then $\theta$ is $C^{\infty}$. We shall show that $\theta$ has a local inverse in a neighborhood of $\mathrm{id}_{V}$. Indeed, let $\left(c_{n}\right)$ be defined by

$$
(1+t)^{1 / 2}=\sum_{n=0}^{\infty} c_{n} t^{n}, \quad(t \in \mathbf{R},|t|<1)
$$

and let

$$
R(u)=\sum_{n=0}^{\infty} c_{n} u^{n}, \quad(u \in E,\|u\|<1) .
$$

It can be easily proved that $\theta(R(u))=\mathrm{id}_{V}+u$.

Recall that $S(x)=B^{-1} \circ\left(S_{2}(x)\right)$ so $S$ maps $\theta$ into $E$ and enjoys the same differentiability properties as $S_{2}$. Thus $S$ is $C^{k}$ in $\theta$ and $C^{1+k}$ in $\theta \backslash\{0\}$ and

$$
\lim _{x \rightarrow 0}\|x\| D^{1+k} S(x)=0 \text {. }
$$

In particular, $S$ is continuous. Choose a neighborhood $U^{\prime}$ of 0 in $\theta$ such that $\|S(x)\|<1$ whenever $x \in U^{\prime}$, and put

$$
A(x)=R(S(x))=\sum_{n=0}^{\infty} c_{n}(S(x))^{n}, \quad x \text { in } U^{\prime} .
$$


From (19a) and Leibnitz' formula, we see that $A$ is $C^{k}$ in $U^{\prime}$ and $C^{1+k}$ in $U^{\prime} \backslash\{0\}$, and

$$
\lim \|x\| D^{1+k} A(x)=0 \text { as } x \rightarrow 0 .
$$

Let $\psi(x)=A(x) x$, then (20) and Theorem 2 give

$$
\psi \text { is } C^{1+k} \text { in } U^{\prime} \text {. }
$$

Now $D \psi(x) h=A(x) h+(D A(x) h) x$, hence

$$
D \psi(0)=A(0)=\mathrm{id}_{V} .
$$

By the inverse mapping theorem, $\psi$ has a local inverse $\varphi: U \leadsto U^{\prime}$ and by $\psi(0)=0,(21)$ and $(22)$,

$$
\varphi \text { is } C^{1+k} \text { in } U, \varphi(0)=0, \quad D \varphi(0)=\mathrm{id}_{V} .
$$

With $h$ in $E$ and $x, y$ in $V$ we have

$$
B(h(x)) h(y)=B(h \circ h(x)) y
$$

hence

$$
B(\psi(y)) \psi(y)=B(A(y) y) A(y) y=B(A(y) \circ A(y) y) y .
$$

But $A(y) \circ A(y)=\theta(A(y))=\mathrm{id}_{V}+S(y)=F(y)$, hence by (18)

$$
B(\psi(y)) \psi(y)=B(F(y) y) y=f(y) .
$$

Substituting $y=\varphi(x)$ and $x=\psi(y)$, we have

$$
f(\varphi(x))=B x^{(2)}=\frac{1}{2} D^{2} f(0) x^{(2)}
$$

as desired.

It remains only to prove that $\varphi$ is $C^{2+k}$ in $U \backslash\{0\}$. Note that in addition to (19a) we also have

$$
S \text { is } C^{2+k} \text { in } \theta \backslash\{0\}
$$

and it follows easily that $A, \psi$ and hence $\varphi$ is $C^{2+k}$ away from 0 . This completes the proof of Theorem 3 .

When the first version of this paper was submitted, the referee kindly called our attention to Tromba's paper [8]. A study of Tromba's paper (loc. cit.) enabled us to arrive at a noteworthy variant to Theorem 3 in the case $V$ is an almost inner product space [8]. Roughly speaking, if $V$ is a Banach space carrying an almost inner product and a smooth duality mapping, then Theorem 3 holds with 0 , weak nondegenerate critical point in the sense of Tromba [8]. Details will appear elsewhere.

\section{REFERENCES}

1. R. Abraham and J. Robbin, Transversal mappings and flows, Benjamin, New York, 1967.

2. Dăng Dinh Áng and Vũ Trọng Tuấn, An elementary proof of the Morse-Palais lemma for Banach spaces, Proc. Amer. Math. Soc. 39 (1973), 642-644.

3. N. Kuiper, $C^{2}$-functions near nondegenerate critical points unpublished manuscript.

4. R. S. Palais, Morse theory on Hilbert manifolds, Topology 2 (1963), 240-299.

5. __ The Morse lemma on Banach spaces, Bull. Amer. Math. Soc. 75 (1969), 968-971. 
6. A. J. Tromba, The Morse lemma on Banach spaces, Proc. Amer. Math. Soc. 34 (1972), 396-402.

7. __ The Morse lemma on arbitrary Banach spaces, Bull. Amer. Math. Soc. 79 (1973), 85-86.

8. __ Almost Riemannian structures on Banach manifolds: The Morse lemma and the Darboux theorem, Canad. J. Math. 38 (1976), 640-652.

9. H. Whitney, Differentiability of the remainder term in Taylor's formula, Duke Math. J. 10 (1943), 153-158.

Department of Mathematics, School of Education, Ho Chi Minh CtTy, Vietnam

Department of Mathematics, The University, Ho Chi Minh City, Vietnam 